\title{
Direction selectivity in the visual system of the zebrafish larva
}

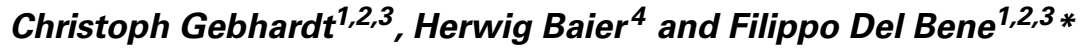 \\ 1 Institut Curie, Centre de Recherche, Paris, France \\ ${ }^{2}$ CNRS UMR 3215, Paris, France \\ ${ }^{3}$ INSERM U934, Paris, France \\ ${ }^{4}$ Department Genes - Circuits - Behavior, Max Planck Institute of Neurobiology, Martinsried, Germany
}

\section{Edited by:}

German Sumbre, Ecole Normale Superieure, France

\section{Reviewed by:}

Carlos D. Aizenman, Brown University, USA

Klaus-Peter Hoffmann, University

Bochum, Germany

\section{*Correspondence:}

Filippo Del Bene, Institut Curie, Centre de Recherche, Batiment. BDD, 11-13 Rue Pierre et Marie Curie, 75005 Paris, France

e-mail: filippo.del-bene@curie.fr
Neural circuits in the vertebrate retina extract the direction of object motion from visual scenes and convey this information to sensory brain areas, including the optic tectum. It is unclear how computational layers beyond the retina process directional inputs. Recent developmental and functional studies in the zebrafish larva, using minimally invasive optical imaging techniques, indicate that direction selectivity might be a genetically hardwired property of the zebrafish brain. Axons from specific direction-selective (DS) retinal ganglion cells appear to converge on distinct laminae in the superficial tectal neuropil where they serve as inputs to DS postsynaptic neurons of matching specificity. In addition, inhibitory recurrent circuits in the tectum might strengthen the DS response of tectal output neurons. Here we review these recent findings and discuss some controversies with a particular focus on the zebrafish tectum's role in extracting directional features from moving visual scenes.

Keywords: visual system, direction selectivity, zebrafish, optic tectum, neural circuits

\section{INTRODUCTION}

Extracting motion information from a visual scene is a key ability of most visual systems throughout the animal kingdom. Moving objects change their position over time in reference to the animal and thus project onto the retina as both spatial and temporal patterns of varying light intensities. With regard to motion detection, an important parameter that can be extracted from these patterns is the direction of a moving stimulus. This information is of vital importance for specific behaviors such as prey capture, collision avoidance, or escape from a predator.

Detailed information has been gathered in insects and mammals about motion processing, but studies were mostly restricted to the computations performed by the sensory surface, i.e., small retinal circuits (Elstrott and Feller, 2009; Borst and Euler, 2011; Wei and Feller, 2011). How direction-selective (DS) is attained and processed by higher brain areas is less evident. Studies addressing this question in the mammalian visual cortex have often investigated either single neurons by electrophysiology or columns of many hundred or thousand of cells by optical imaging, thus lacking the resolution necessary to ask circuit-level questions (Bonhoeffer and Grinvald, 1991; Chapman etal., 1996; Priebe et al., 2010).

The optic tectum of larval zebrafish is an excellent brain structure to study motion processing at a higher circuit-level. The tectum is the main retinorecipient brain region and homologous to the superior colliculus in mammals. Sitting at the surface of the brain, it is easily accessible to a wide variety of techniques, including electrophysiology, laser ablations, optogenetics, and optical imaging. In addition to receiving a majority of retinal afferents, the tectum is an integrator of sensory information from multiple modalities (Meek, 1983; Vanegas and Ito, 1983). Main areas of the tectum can be histologically distinguished. The stratum periventriculare (SPV) contains the cell bodies of most tectal neurons (periventricular neurons, PVNs) whereas the synaptic neuropil area contains the PVNs' dendrites and axons as well as the axons of retinal ganglion cells (RGCs). The tectal neuropil is a precisely laminated structure within which the RGC axons mostly target the superficial layers (Xiao et al., 2005): the stratum opticum (SO), right beneath the basement membrane, and the stratum fibrosum et griseum superficiale (SFGS). Classical Golgi studies in adult goldfish and genetic single-cell labeling in larval zebrafish revealed that the PVNs have a single dendritic shaft that extends into the tectal neuropil, often crossing multiple layers (Vanegas et al., 1974; Meek and Schellart, 1978; Scott and Baier, 2009; Nevin et al., 2010; Robles et al., 2011).

Importantly, zebrafish are also genetically accessible rendering them well suited for functional studies of the visual system that require targeting of protein-based indicators to genetically identified subpopulations of neurons. This opens up the exciting possibility of studying DS processing across specific neuronal populations, often with single-cell resolution.

\section{DEVELOPMENT OF DS IN THE OPTIC TECTUM APPEARS TO BE GENETICALLY HARDWIRED}

The anatomical and morphological development of the zebrafish larval visual system has been investigated in great detail (e.g., Stuermer, 1988). Between 34 and 48 hours post fertilization (hpf) retinal axons leave the retina and start invading the tectal neuropil. By $72 \mathrm{hpf}$, retinal axons have sparsely innervated the entire tectum and begin to form terminations at their topographically correct targets. At around the same time, the lens has developed to produce a focused image within the photoreceptor layer of the retina 
(Easter and Nicola, 1996). After tectal coverage is achieved, dendritic arbors undergo remodeling until a relatively stable state is reached around 7 days post fertilization (dpf). The laminar development of retinotectal wiring seems to be largely independent of externally evoked visual activity. Activity-dependent mechanisms, however, influence the refinement of the RGC arbors that form the visuotopic map (Stuermer et al., 1990; Gnuegge et al., 2001; Hua et al., 2005; Smear et al., 2007; Nevin et al., 2008; Fredj et al., 2010).

Extraction of directional information from a visual scene requires that DS neurons exhibit an asymmetric response to visual stimuli that move in the preferred vs. the opposite (null) direction. This functional asymmetry must ultimately be a consequence of an asymmetry in wiring, regulation of synaptic strengths, or dendritic conductance. How does this asymmetry come about during development? Several possibilities have been proposed. For one, it could be that this asymmetry of DS circuits is genetically hardwired, for instance by cell-surface molecular cues that act upon dendrite or synapse distribution and are expressed very early in visual system development. It is also possible that DS circuits show initially non-asymmetric responses and are subsequently biased in one direction by activity-dependent mechanisms. Of course, genetic hardwiring and activity-based mechanisms might also act in concert to shape the final DS response of neurons of the visual system.

In a landmark study, Niell and Smith (2005) used in vivo $\mathrm{Ca}^{2+}$ imaging with the synthetic $\mathrm{Ca}^{2+}$ indicator OGB1-AM for an initial functional description of the entire tectal cell population during development. Among other visual parameters, the authors also examined DS in the tectum. They reported that a large proportion of tectal cells were already DS as early as $72 \mathrm{hpf}$ and DS reached nearly mature levels after $78 \mathrm{hpf}$. This is perhaps surprising considering that during that time window the very first retinal axons have barely reached their termination zones in the tectum and retinotectal circuits are still undergoing thorough refinement. Furthermore, zebrafish larvae that were reared completely in the dark showed normal DS responses, which were indistinguishable from larvae reared under default light-dark cycle conditions.

The latter finding is not consistent with a study in Xenopus tadpoles (Engert et al., 2002). This paper reported that DS of tectal cells was not apparent at early developmental stages but extensive training with a moving stimulus was able to induce DS responses in a few recorded tectal neurons, suggesting an experience-dependent mode of DS development. This discrepancy between zebrafish and Xenopus could be due to a true species difference as others (Podgorski et al., 2012) have also found DS plasticity after visual training in tadpoles. However, it might also be possible that in tadpoles, DS of tectal cells is present at early stages and repeated training generated short-lasting single neuron and/or network connectivity changes that obscured the initially hardwired tuning of the recorded tectal cells.

Niell and Smith's findings were, however, largely confirmed and extended, by a later study (Ramdya and Engert, 2008). Normally, retinal projections to the tectum are completely crossed, i.e., tectal neurons are monocular. By surgically removing a single tectal lobe the authors partially re-routed the retinal projection to the ipsilateral tectum, thereby generating a few binocularly innervated tectal cells (i.e., neurons that responded to inputs from both eyes). They found that these binocular cells showed the same DS response to moving stimuli when these were presented to either eye. Furthermore, depriving the animals from any externally evoked visual activity by rearing them in the dark, showed again no difference in the development of the observed binocular DS compared to light-reared larvae. This is in agreement with the experience-independent DS development observed earlier by Niell and Smith (2005). Furthermore, since it is also unlikely that correlated intrinsic activities between the two eyes occur, this might indicate that the development of direction selectivity in zebrafish is, in addition to being experience-independent, also independent of spontaneously generated retinal waves (Wong et al., 1993).

Taken together the available evidence suggests that, at least in zebrafish, direction selectivity is established at the earliest stage measurable and develops independently of activity in the visual system.

\section{TECTUM-INTRINSIC COMPUTATION OF DIRECTION SELECTIVITY}

Functional models of DS differ mainly in how excitatory or inhibitory input currents are tuned (i.e., to the preferred vs. the null-direction) and then integrated in time to finally give rise to tectal DS outputs. In the zebrafish, there is evidence for two different DS mechanisms being implemented. For one, DS could be predominantly computed by retinal circuits, which then would drive postsynaptic DS neurons directly via direction-tuned excitatory inputs. Alternatively, excitatory retinal input might show rather weak DS and subsequent tectal recurrent inhibition, tuned to the null-direction, might shape the final PVN response in the preferred direction of the stimulus. Both of these mechanisms are not mutually exclusive and might be implemented in a complementary fashion in retina and tectum (Figure 1).

In the above-mentioned study, Ramdya and Engert (2008) found evidence for a null-inhibition mechanism in the zebrafish tectum. By using the surgically induced binocular retinotectal circuit they addressed if direction selectivity is computed in the retina and then projected into pre-specified tectal modules or if, alternatively, tectal inhibitory circuits perform this computation. Two lines of evidence suggested that the latter mechanism is at work. First, the authors performed an experiment in which they displayed a visual stimulus consisting of two stationary spots separated in time and jumping between different visual field positions of one eye. These two stationary spots are seen as apparent motion and elicit a DS response in a subset of the recorded tectal neurons. Showing these two spots with a slight delay to the left and right eye of fish with binocular input to the tectum, should not elicit any DS response if direction selectivity relies purely on retinal computation. However, the authors observed that some binocular tectal cells were showing a response to an apparent-motion stimulus that was comparable to the stimulus applied to the contralateral eye alone. Second, a pharmacological block of tectal inhibition by injection of bicuculline in the tectum led to a loss of DS in most of the tectal neurons under normal conditions. This was due to the response to the null-direction being strongly increased after drug injection. 


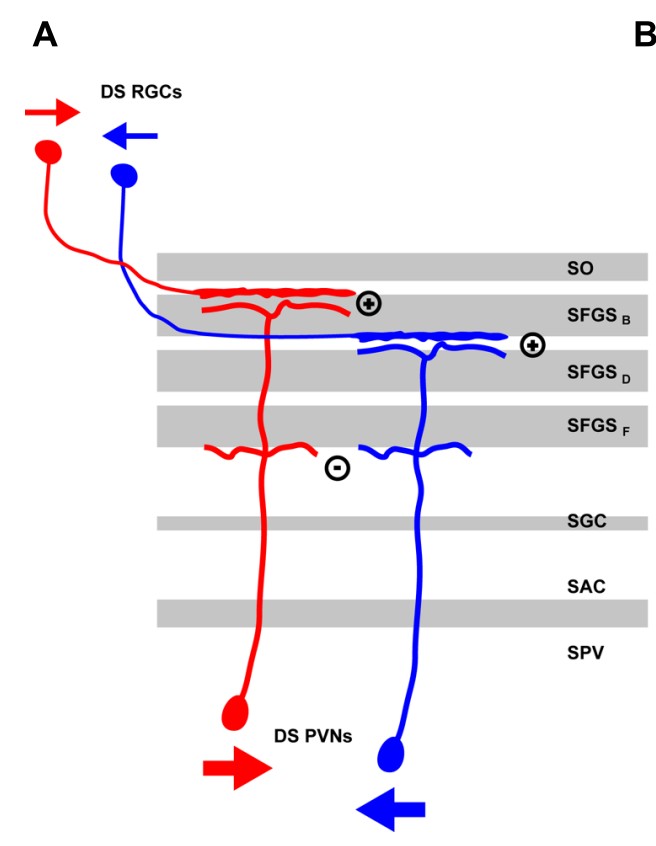

FIGURE 1 | Mechanisms for direction selectivity computation in the zebrafish larva tectum. (A) Direction information from a moving stimulus is extracted by retinal circuits and transferred by RGC axons, which are specific for the stimulus direction (small red or blue arrows), to distinct laminae in the tectal neuropil. The retinal arbors are then targeted by the distal arbors of PVNs in their respective laminae thus acquiring direction specificity themselves. Heterotypic connections between proximal PVN arbors might lead to reciprocal inhibition thus sharpening DS PVN response to a moving stimulus in a specific direction (large red or blue arrows). (B) A DS PVN (blue) receives excitatory inputs from one or more non-DS RGCs in the tectal

neuropil. In addition, it receives intratectal inhibitory input from an interneuron

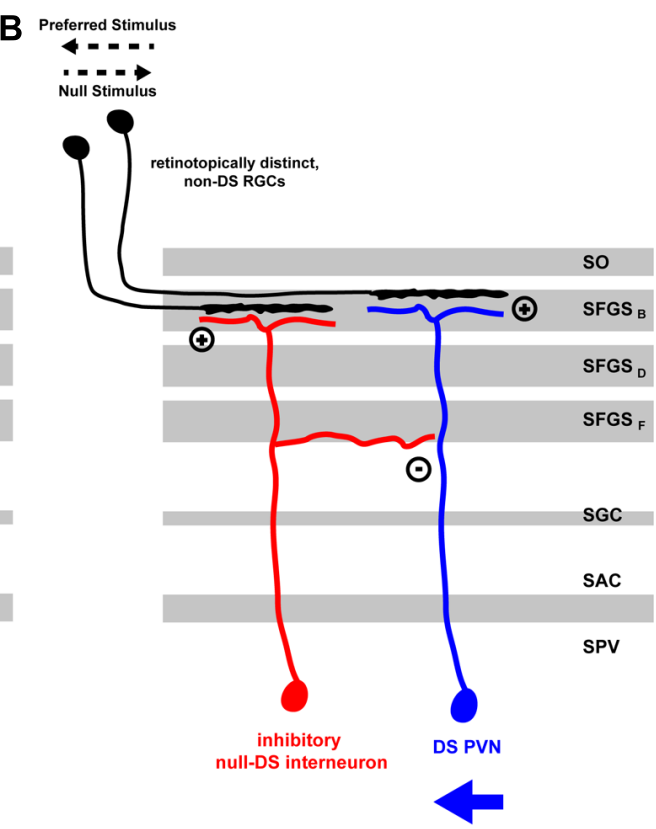

(red) that is retinotopically positioned on the side of the DS PVN facing the preferred stimulus direction. Thus, a moving stimulus in the preferred direction (black pointed arrow) elicits excitatory currents in the retina that excite DS PVNs. Currents from the inhibitory interneurons arrive later and do not interfere with the PVNs activity state. Moving stimuli in the null-direction, however, elicit inhibitory currents in the tectal interneuron, which arrive first at the DS PVN thus blocking any subsequent excitatory currents the DS PVN might receive from the retina. DS, direction-selective; $R G C$, retinal ganglion cell; PVN, periventricular neuron; SAC, stratum album centrale; SFGS (B-F), sublaminae of stratum fibrosum et griseum superficiale; SGC, stratum griseum centrale; SO, stratum opticum; SPV, stratum periventriculare.
Taken together, these results provided evidence for a tectal DS computing mechanism involving strong recurrent inhibition tuned to the null-direction rather than direct retinal excitatory currents. It is unclear, however, if the artificially altered circuit is indeed indicative of how direction selectivity is computed under normal, unaltered conditions or if the apparent-motion stimulus is at all suited for investigating feature extraction from a moving visual scene. For instance, if the retinal inputs to the recorded tectal cells are already DS, then activation by the apparent-motion stimulus, even though it may not be the optimal stimulus, will elicit a DS response in the postsynaptic cell. This response can look deceptively similar to a tectum-intrinsic, de novo DS computation.

In a follow-up paper, Grama and Engert (2012) analyzed the contribution of excitatory and inhibitory currents to tectal DS in more detail. By patching a random set of tectal neurons in the SPV, they found that excitatory input currents, supposedly originating from the retina, were correlated but not tuned to the direction of the stimulus motion, as measured by counting the spikes evoked by a moving bar. However, inhibitory currents, presumably coming from tectal inhibitory interneurons, were inversely correlated with the direction of motion, i.e., they were biased for the null-direction. Furthermore, the authors observed latencies in the millisecond range between excitatory and inhibitory currents. Inhibitory currents preceded the excitatory ones in the null-direction (median $=39 \mathrm{~ms}$ ) and vice versa in the preferred direction (median $=157 \mathrm{~ms}$ ). Based on this evidence, the authors suggested a model in which tectal DS responses are computed from non-DS retinal inputs by tectal recurrent inhibition. For such a mechanism to work, they postulated the existence of a special type of tectal interneuron, which, similar to the starburst amacrine cell in the retina (Fried et al., 2002), is responding to moving stimuli in the null-direction and is asymmetrically connected to DS tectal output neurons (Figure 1). This interneuron type should be positioned on the side of the DS cell that represents the preferred direction along the corresponding axis of the retinotopic map. In this configuration, stimuli coming from the null-direction will selectively suppress the response of the output cell. While plausible, there is currently no evidence for the existence of such an asymmetrically organized circuit in the zebrafish tectum.

\section{TECTAL PROCESSING OF DIRECTION-SELECTIVE RETINAL INPUTS}

Differing from an exclusively tectum-intrinsic mechanism for direction computation, two recent studies showed that RGC inputs are already tuned to stimulus direction when they reach the tectum. In the first study, Meyer and colleagues expressed the genetically encoded $\mathrm{Ca}^{2+}$ indicator SypGCaMP3, driven by 
the $i s l 2 b$ promoter, in almost all retinal synapses terminating in the retinorecipient layers of the tectum (Nikolaou et al., 2012). By statistical analysis of activity distribution maps from stacks of several animals over several experimental trials, they found three major RGC DS input clusters in the tectal neuropil, one caudal-to-rostral and two different rostral-to-caudal directed clusters with a down-up or up-down DS component respectively. These DS clusters match the response tuning profiles of the previously reported DS-RGCs in goldfish (Maximov et al., 2005). In the tectum, these inputs were segregated superficially in two discrete layers of the SFGS. Caudal-to-rostral-tuned inputs were distributed more superficially in the tectal neuropil than inputs of the other directions. Notably, these layers are preferentially innervated by RGCs that have bistratified dendrites in the ON and OFF sublayers of the inner plexiform layer of the retina (Robles et al., 2013). RGCs of this class have been shown to be DS in several other vertebrates, including birds, mammals, and fish.

Furthermore, Nikolaou etal. (2012) reported that retinal synaptic inputs responding to caudal-to-rostral motion predominated quantitatively over those responding to rostral-to-caudal motion, which is consistent with previous studies (Maximov et al., 2005; Niell and Smith, 2005). Moreover, the authors found two clusters of orientation-selective (OS) presynaptic inputs (horizontal and vertical motion in both directions) that spanned several laminae in the middle layers of the neuropil and were well separated from DS inputs in the superficial neuropil. Surprisingly, they observed also a retinotopic bias of the observed DS and OS clusters. The DS inputs were mostly confined to the posterior half of the tectum while the two OS clusters were distributed anteriorly and posteriorly, respectively. It is currently unclear if these distributions reflect the existence of topographically restricted RGC populations, retinotopic differences in the retinal circuits or presynaptic modulation within the tectum. Considering that these cumulative imaging data were highly processed and thresholded, it is also possible that synapse density of DS cells and thus SypGCaMP3 expression accounted for the observed topographic differences.

The most comprehensive study of DS in the zebrafish retinotectal system so far was recently presented by Bollmann and colleagues (Gabriel et al., 2012). From an enhancer-trap screen, they identified two Gal4-VP16 transgenic lines that labeled subsets of DS interneurons in the tectum. $\mathrm{Tg}(\mathrm{Oh}: \mathrm{G3})$ drives expression of UAS (upstream activation sequence)-linked reporter genes mostly in rostral-to-caudal-tuned cells, whereas $\mathrm{Tg}(\mathrm{Oh}: \mathrm{G} 4)$ labels caudalto-rostral-tuned cells. In addition to differences in DS, these two subsets of tectal neurons also differ morphologically. While both are bistratified, they have their distal dendritic arbor in different layers of the tectal neuropil.

Similar to Nikolaou et al. (2012), Gabriel et al. (2012) reported three main types of presynaptic DS inputs (one caudal-to-rostral cluster and two rostral-to-caudal ones) and observed that each targets one specific lamina in the tectal neuropil. In a series of elegant experiments, Gabriel et al. (2012) showed that functionally identified postsynaptic neurons had their dendritic arbors specifically in the very same laminae in the neuropil as the matching DS RGC inputs, lending further weight to their mapping of DS retinal inputs.
While the two studies by Nikolaou et al. (2012) and Gabriel et al. (2012) converge on the same broad conclusions they differ in important details, which appear irreconcilable at first glance. Gabriel et al. (2012) observed one rostral-to-caudal-tuned cluster (with both up-down and down-up DS component) that was situated more superficially in the tectal neuropil than the caudalto-rostral-tuned cluster. This is the inverse of what Nikolaou and colleagues reported. How can this apparent discrepancy be explained? The precise layout of the laminar distribution of retinal inputs in the tectal neuropil might offer a solution. In a parallel set of studies, utilizing brain bow labeling of RGC axons, Robles et al. (2013) reported that the zebrafish retinotectal neuropil is composed of at least ten laminae. The SO is subdivided into two layers, SO1 and SO2, while the SFGS contains six distinct layers, SFGS1 through SFGS6. Each of the retinotectal layers harbors a complete retinotopic map and is innervated by a distinct combination of RGC types (Robles et al., 2013). In this new scheme, Gabriel et al.'s DS inputs might, for instance, be localized to SO2, SFGS1 and maybe SFGS2, while Nikolaou et al.'s might be in SFGS1 and SFGS2. The two studies would then unequivocally agree that one of the ten layers, most likely SFGS1, is sensitive to caudal-to-rostral direction.

The reason for the differences in both studies could be that weak rostral-to-caudal oriented signals (i.e. rostral-to-caudal signals found in SO2 and SFGS2, respectively) might have been difficult to record: In order to isolate the presynaptic activity in the tectum, Gabriel et al. (2012) used pan-neuronal GCaMP3-expression and subsequent pharmacological blockage of glutamatergic transmission in tectal cells. This approach could have lead to a high intensity background impeding the detection of weak clusters. By contrast, the genetic targeting and/or expression levels of SypGCaMP3 in the study by Nikolaou et al. (2012) might not have been sufficient to reveal all existing retinal laminae. Furthermore, the threshold for identifying DS input signals were set differently in the two studies. This choice might also have contributed to the observed differences.

In summary, a scheme that assumes the existence of three presynaptic layers in the superficial third of the neuropil with alternating DS, a caudal-to-rostral lamina sandwiched between two rostral-to-caudal oriented ones (possibly each of the latter containing two distinct sub-clusters with a down-up or updown DS component, respectively), might explain the available data.

Furthermore, Gabriel et al. (2012) reported that excitatory inputs, likely from RGCs, determine the DSof at least some tectal cell types. This is in agreement with Nikolaou et al. (2012), but appears to contradict the conclusions of Grama and Engert (2012) who did not find DS-tuned excitatory inputs but emphasized rather the importance of inversely DS-tuned inhibitory intra-tectal currents. Gabriel et al. (2012) also report that the two different types of bistratified DS tectal neurons are GABAergic, inhibitory interneurons. Thus, they suggest that a feed-forward, null-direction inhibition via the proximal dendritic arbors of the cells might serve as a means to fine-tune the tectum's output. It is still possible that some types of tectal cells are mainly driven by DS excitatory input, whereas others are controlled by DS-tuned inhibitory inputs. 


\section{CONCLUSION AND DIRECTIONS FOR FUTURE RESEARCH}

DS neurons are found in several regions along the visual pathway, including retina, tectum, and cortex. It is important to understand how neurons acquire DS characteristics at each of these stages. Studies in zebrafish have revealed that DS is hardwired and can develop independently of patterns of activity. In the tectum, DS retinal inputs terminate in the tectal neuropil in specific laminae, where they form connections with the lamina-restricted dendrites of tectal interneurons. This feed-forward mode of DS wiring is reminiscent of the so-called "labeled lines" that are found in other sensory systems (Kauer and White, 2001). Evidence for the contribution of tectal recurrent connections, especially inhibitory ones, is less clear. If it exists, it might contribute to sharpening the response of DS output neurons.

In conclusion, it seems to us that, for a complete understanding of DS computation, additional genetic markers for functionally

\section{REFERENCES}

Bonhoeffer, T., and Grinvald, A. (1991). Iso-orientation domains in cat visual cortex are arranged in pinwheel-like patterns. Nature 353, 429-431. doi: 10.1038/353429a0

Borst, A., and Euler, T. (2011). Seeing things in motion: models, circuits, and mechanisms. Neuron 71, 974-994. doi: 10.1016/j.neuron.2011. 08.031

Chapman, B., Stryker, M. P., and Bonhoeffer, T. (1996). Development of orientation preference maps in ferret primary visual cortex. J. Neurosci. 16, 6443-6453.

Easter, J., and Nicola, G. N. (1996). The development of vision in the zebrafish (Danio rerio). Dev. Biol. 180, 646-663. doi: 10.1006/dbio. 1996.0335

Elstrott, J., and Feller, M. B. (2009). Vision and the establishment of direction-selectivity: a tale of two circuits. Curr. Opin. Neurobiol. 19, $293-$ 297. doi: 10.1016/j.conb.2009.03.004

Engert, F., Tao, H. W., Zhang, L. I., and Poo, M. (2002). Moving visual stimuli rapidly induce direction sensitivity of developing tectal neurons. Nature 419, 470-475. doi: 10.1038/nature00988

Fredj, N. B., Hammond, S., Otsuna, H., Chien, C.-B., Burrone, J., and Meyer, M. P. (2010). Synaptic activity and activity-dependent competition regulates axon arbor maturation, growth arrest, and territory in the retinotectal projection. J. Neurosci. 30, 10939-10951. doi: 10.1523/ JNEUROSCI.1556-10.2010

Fried, S. I., Münch, T. A., and Werblin, F. S. (2002). Mechanisms and circuitry underlying directional selectivity in the retina. Nature 420, 411-414. doi: 10.1038 /nature 01179
Gabriel, J. P., Trivedi, C. A., Maurer, C. M., Ryu, S., and Bollmann, J. H. (2012). Layer-specific targeting of direction-selective neurons in the zebrafish optic tectum. Neuron 76, 1147-1160. doi: 10.1016/ j.neuron.2012.12.003

Gnuegge, L., Schmid, S., and Neuhauss, S. C. F. (2001). Analysis of the activity-deprived zebrafish mutant macho reveals an essential requirement of neuronal activity for the development of a fine-grained visuotopic map. J. Neurosci. 21, 3542-3548.

Grama, A., and Engert, F. (2012). Direction selectivity in the larval zebrafish tectum is mediated by asymmetric inhibition. Front. Neural Circuits 6:59. doi: 10.3389/fncir.2012.00059

Hua, J. Y., Smear, M. C., Baier, H., and Smith, S. J. (2005). Regulation of axon growth in vivo by activity-based competition. Nature 434, 1022-1026. doi: 10.1038/nature03409

Huberman, A. D., Wei, W., Elstrott, J., Stafford, B. K., Feller, M. B., and Barres, B. A. (2009). Genetic identification of an On-Off direction- selective retinal ganglion cell subtype reveals a layer-specific subcortical map of posterior motion. Neuron 62, 327-334. doi: 10.1016/j.neuron.2009.04.014

Kauer, J. S., and White, J. (2001). Imaging and coding in the olfactory system. Ann. Rev. Neurosci. 24, 963979. doi: 10.1146/annurev.neuro.24. 1.963

Maximov, V., Maximova, E., and Maximov, P. (2005). Direction selectivity in the goldfish tectum revisited. Ann. N. Y. Acad. Sci. 1048, 198-205. doi: 10.1196/annals.1342.018

Meek, J. (1983). Functional anatomy of the tectum mesencephali of the goldfish. An explorative analysis of the functional implications of the

identified types of DS neurons are needed, not only in the tectum but also in the retina (Huberman et al., 2009; Gabriel et al., 2012). It will be productive to trace the connections of the different types of DS-RGCs from the retina to the tectal layers and identify their postsynaptic partners. Future research should also elucidate how DS computation is used in behavioral contexts, i.e., how DS information is transferred to motor centers and used to generate oriented behavior toward prey or away from predators. The zebrafish tectum, as a prominent center for sensorimotor transformation in an optically and genetically accessible organism, will be an excellent place to investigate these fundamental questions of systems neuroscience.

\section{ACKNOWLEDGMENTS}

We like to thank Johann Bollmann, Thomas Auer and Alessandro Filosa for their comments on the manuscript. Michael Orger provided valuable insight and advice on imaging.

laminar structural organization of the tectum. Brain Res. 287, 247-297.

Meek, J., and Schellart, N. A. (1978) A Golgi study of goldfish optic tectum. J. Comp. Neurol. 182, 89-122. doi: 10.1002/cne.901820107

Nevin, L. M., Robles, E., Baier, H., and Scott, E. K. (2010). Focusing on optic tectum circuitry through the lens of genetics. BMC Biol. 8:126. doi: 10.1186/1741-7007-8-126

Nevin, L. M., Taylor, M. R., and Baier, H. (2008). Hardwiring of fine synaptic layers in the zebrafish visual pathway. Neural Dev. 3, 36. doi: 10.1186/17498104-3-36

Niell, C. M., and Smith, S. J. (2005). Functional imaging reveals rapid development of visual response properties in the zebrafish tectum. Neuron 45, 941-951. doi: 10.1016/ j.neuron.2005.01.047

Nikolaou, N., Lowe, A. S., Walker, A. S., Abbas, F., Hunter, P. R., Thompson, I. D., et al. (2012). Parametric functional maps of visual inputs to the tectum. Neuron 76, 317-324. doi: 10.1016/j.neuron.2012.08.040

Podgorski, K., Dunfield, D., and Haas, K. (2012). Functional clustering drives encoding improvement in a developing brain network during awake visual learning. PLoS Biol. 10:e1001236. doi: 10.1371/journal. pbio. 1001236

Priebe, N. J., Lampl, I., and Ferster, D. (2010). Mechanisms of direction selectivity in cat primary visual cortex as revealed by visual adaptation. J. Neurophysiol. 104, 2615-2623. doi: 10.1152/jn.00241.2010

Ramdya, P., and Engert, F. (2008). Emergence of binocular functional properties in a monocular neural circuit. Nat. Neurosci. 11, 1083-1090. doi: 10.1038/nn.2166
Robles, E., Filosa, A., and Baier, H. (2013). Precise lamination of retinal axons generates multiple parallel input pathways in the tectum. J. Neurosci. 33, 5027-5039. doi: 10.1523/JNEUROSCI.4990-12. 2013

Robles, E., Smith, S. J., and Baier, H. (2011). Characterization of genetically targeted neuron types in the zebrafish optic tectum. Front. Neural Circuits 5:1. doi: 10.3389/fncir. 2011.00001

Scott, E. K., and Baier, H. (2009). The cellular architecture of the larval zebrafish tectum, as revealed by Gal4 enhancer trap lines. Front. Neural Circuits 3:13. doi: 10.3389/ neuro.04.013.2009

Smear, M. C., Tao, H. W., Staub, W., Orger, M. B., Gosse, N. J., Liu, Y., et al. (2007). Vesicular glutamate transport at a central synapse limits the acuity of visual perception in zebrafish. Neuron 53, 65-77. doi: 10.1016/j.neuron.2006.12.013

Stuermer, C. A. (1988). Retinotopic organization of the developing retinotectal projection in the zebrafish embryo. J. Neurosci. 8, 4513-4530.

Stuermer, C. A., Rohrer, B., and Münz, H. (1990). Development of the retinotectal projection in zebrafish embryos under TTX-induced neuralimpulse blockade. J. Neurosci. 10, 3615-3626.

Vanegas, H., and Ito, H. (1983). Morphological aspects of the teleostean visual system: a review. Brain Res. 287, 117-137. doi: 10.1016/01650173(83)90036-X

Vanegas, H., Laufer, M., and Amat, J. (1974). The optic tectum of a perciform teleost I. General configuration and cytoarchitecture. $J$. 
Comp. Neurol. 154, 43-60. doi: 10.1002/cne.901540104

Wei, W., and Feller, M. B. (2011). Organization and development of direction-selective circuits in the retina. Trends Neurosci. 34, 638-645. doi: 10.1016/j.tins.2011.08.002

Wong, R. O. L., Meister, M., and Shatz, C. J. (1993). Transient period of correlated bursting activity during development of the mammalian retina. Neuron 11 ,
923-938. doi: 10.1016/0896-6273 (93) 90122-8

Xiao, T., Roeser, T., Staub, W., and Baier, H. (2005). A GFPbased genetic screen reveals mutations that disrupt the architecture of the zebrafish retinotectal projection. Development 132, 2955-2967. doi: 10.1242/dev.01861

Conflict of Interest Statement: The authors declare that the research was conducted in the absence of any commercial or financial relationships that could be construed as a potential conflict of interest.

Received: 08 March 2013; paper pending published: 05 April 2013; accepted: 28 May 2013; published online: 18 June 2013.

Citation: Gebhardt C, Baier H and Del Bene F (2013) Direction selectivity in the visual system of the zebrafish larva. Front. Neural Circuits 7:111. doi: 10.3389/fncir.2013.00111

Copyright () 2013 Gebhardt, Baier and Del Bene. This is an open-access article distributed under the terms of the Creative Commons Attribution License, which permits use, distribution and reproduction in other forums, provided the original authors and source are credited and subject to any copyright notices concerning any third-party graphics etc. 